\title{
Early diagnosis and surgical management of heterotopic pregnancy allows us to save the intrauterine pregnancy
}

\author{
Gaspare Cucinella ${ }^{1}$, Giuseppe Gullo ${ }^{1}$, Andrea Etrusco ${ }^{1}$, Erika Dolce ${ }^{2}$, Silvia Culmone ${ }^{1}$, \\ Giovanni Buzzaccarini ${ }^{3}$ \\ ${ }^{1}$ Obstetrics and Gynaecology Department, Villa Sofia Cervello Hospital, University of Palermo, Italy \\ ${ }^{2}$ Family Psychotherapy Academy, Palermo, Italy \\ ${ }^{3}$ Department of Women's and Children's Health, Gynaecology and Obstetrics Clinic, University of Padua, Italy
}

\begin{abstract}
Heterotopic pregnancy is a rare but intriguing disease, which poses a high risk for pregnant women and for intrauterine pregnancy. Clinically, it is mainly characterized by pain and vaginal bleeding. $\beta$-hCG serum dosage is used to detect the pregnancy, but transvaginal ultrasound is needed to diagnose heterotopic pregnancy. From all the risk factors, the assisted reproductive treatments represent the most important, especially when multiple embryos are transferred. Patients with a heterotopic pregnancy are at risk of having a spontaneous or medically induced abortion, and for this reason appropriate and tailored treatment should be considered, pursuing the optimal risk/benefit ratio. Although conservative treatments are available in the case of only extrauterine pregnancy, such as methotrexate, often the laparoscopic approach seems unavoidable in heterotopic pregnancy. To preserve the intrauterine pregnancy, a conservative treatment is remarkable, both salpingotomy or salpingostomy. In this case report, we present a successful laparoscopic treatment of an extrauterine pregnancy with the prosecution of the intrauterine pregnancy up to the third trimester. In particular, a 25-year-old girl referred to the Villa Sofia Cervello Hospital complaining of abdominopelvic pain and vaginal bleeding for 2 days. The salpingectomy approach was performed, and every anatomical piece was sent for histopathology.
\end{abstract}

Key words: heterotopic pregnancy, salpingectomy, laparoscopy, pregnancy-sparing.

\section{Introduction}

Heterotopic pregnancy refers to the simultaneous presence of intrauterine and ectopic gestational chambers. Epidemiologically it affects about 1 in 15,000/30,000 naturally conceived pregnancies [1], and the risk of heterotopic pregnancy rises from 1 to $3 \%$ for pregnancies that occur through assisted reproductive technologies (ART) [2]. Patients with a heterotopic pregnancy are also more likely to have a spontaneous or medically induced abortion and are 30\% less likely to have a live-birth delivery compared with women with isolated intrauterine pregnancies [3]. Because heterotopic pregnancy is difficult to diagnose, patients affected are at increased risk of tubal rupture, hypovolaemic shock, and maternal or foetal death [1]. Retainment of the dead foetus in abdominal cavity is extremely rare, known as lithopaedion [4]. The known risk factors that contribute to the development of a heterotopic pregnancy that we know of are the following: pelvic inflammatory disease, previous ectopic pregnancies, previous abdominal surgeries involving the fallopian tubes, the use of intrauterine devices, age $>35$ years, frequent use of vaginal douches, smoking, and a positive anamnesis for infertility treated with medically ART $[2,5]$. The greatest risk factor for the development of heterotopic pregnancy is ART. The risk of abdominal pregnancy is up to 8 times greater in women undergoing in vitro fertilization (IVF) compared with the general population [2]. The risk is further increased if IVF is performed in women with tubal pathology and when multiple embryos are transferred [2]. Regarding this case, the benefit of ultrasound-guided embryo transfer to avoid ectopic pregnancy has been investigated, with little success [6]. Moreover, another peculiar ectopic pregnancy location could be that of interstitial pregnancy. Laparoscopic treatment of interstitial pregnancy requires accurate haemostasis due to the high vascularization of the uterine cornua. In this case, a reversible laparoscopic uterine artery occlusion can be performed with success [7]. Other feasible approaches encounter the combination of systemic and local administration of methotrexate with hysteroscopic [8].

Modern biochemical technology, by means of $\beta$-hCG assay and ultrasound, has greatly simplified investigations into the diagnosis of ectopic and/or heterotopic pregnancy. No reasoning made on the sole evaluation

Corresponding author:

Giovanni Buzzaccarini, MD, Gynaecologic and Obstetrics Clinic, Department of Women's and Children's

Submitted: 08.08.2021

Health, University of Padua, via Nicolò Giustiniani 3, 35128, Padua, Italy,

Accepted: 29.08 .2021

e-mail: giovanni.buzzaccarini@gmail.com 
of the growth or decreasing trend of $\beta-h C G$ allows us to give information about the implantation site of the pregnancy. The only role of the $\beta$-hCG dosage is to demonstrate the existence of pregnancy and to predict its initial evolution. The role of ultrasound is to locate the site of the pregnancy [9]. Transvaginal ultrasound allows for a precocity and a diagnostic accuracy far superior to that obtained with the abdominal approach. Although the main purpose of the ultrasound is to demonstrate unequivocally that the gestational chamber is correctly implanted inside the uterus, the study of other possible implantation sites of the pregnancy must be equally fundamental from the first visit and continuing in the visits to follow [9]. Confirming an intrauterine pregnancy via ultrasound or clinically does not exclude the coexistence of an ectopic pregnancy [9]. This makes early diagnosis of heterotopic pregnancy challenging, especially when the patient is asymptomatic, as seen during early ultrasound screening examinations [9]. The signs and symptoms of a heterotopic pregnancy are subtle and often absent on first visits, which can lead to a delay in diagnosis $[5,9]$. When they are present, they more frequently consist of abdominal pains of greater intensity than one might expect. Pain is present in more than $90 \%$ of cases, even when the ectopic implantation pregnancy has not yet caused injury to the affected bowel. Vaginal bleeding is the second most frequent symptom. Severe abdominal pain localized to the lower quadrants is associated with a ruptured ectopic pregnancy, which is a surgical emergency $[5,9,10]$.

A heterotopic pregnancy significantly differs from a normal extrauterine pregnancy due to the importance of preserving the intrauterine pregnancy. For this reason, possible treatments such as methotrexate should be avoided if the intrauterine safety is to be preserved [1]. Regarding the surgery, in the case of tubal pregnancy, the approaches can be both salpingostomy and salpingectomy [11]. However, recent data reported a similarity in terms of intrauterine pregnancy after 24 months and 2-year recurrency of ectopic pregnancy. However, the persistency of trophoblast was $1.8 \%$ for salpingectomy and $12 \%$ for salpingostomy [12].

\section{Case presentation and discussion}

A pregnant 25 -year-old female in the $5^{\text {th }}$ week +4 days of amenorrhoea presented to the obstetrics and gynaecology emergency room with the chief complaints of abdominopelvic pain and vaginal bleeding for 2 days.

On scrutiny: last period 20/11/2020, pregnancy obtained in a natural way, no full-term pregnancy in the past, no history of ectopic pregnancy in the past, 1 voluntary termination of pregnancy 2 years before at 10 weeks of pregnancy followed by revision of the uterine cavity, no intrauterine device inserted, no inflammatory pathologies or other noteworthy ones, no history of previous abdominal surgery, no smoking or drug addiction, the patient did not take any kind of drug, and she only took folic acid for the pregnancy.

On examination, apart from mild tachycardia, other vital parameters were stable. The abdomen was treatable and painless on superficial and deep palpation on all the upper quadrants; pain was evoked on superficial and deep palpation on the lower quadrants, especially in the left iliac fossa. Sore Douglas. Normal and nulliparous external genitalia. Regular vagina and cervix, mobile uterine body not contracted and not painful when mobilized. Ovaries non palpable, painful left adnexal region. Blood traces, and not atypical losses from external genitalia on vaginal exploration.

Transvaginal ultrasound was performed: the uterus had regular echo-structure and slightly increased morphovolumetry, inside, where a eumorphic gestational chamber was visualized, $19 \times 0.9 \mathrm{~mm}$ in size, with the presence of a yolk sac and embryonic echoes, crown rump length $3.4 \mathrm{~mm}$ corresponding to the gestational period, and foetus with foetal heart activity. The right adnexal region was within normal limits. Left adnexal region site of unilocular formation, to refer to corpus luteum of the size of $24 \times 19 \mathrm{~mm}$. Above this formation an anecogneal area with a hyperechoic border was echovisualized, to be referred to as a probable tubal ring. Within this formation the following are highlighted: a small hyperechoic area presenting vascular activity (confirmed by the pulsed Doppler) and a circular formation ascribable to the yolk sac: ascribable to a tubal pregnancy. The presence of a modest free fluid flap in the Douglas cable with dimensions of $61 \times 15 \times 51 \mathrm{~mm}$. Morrison pocket free from fluid flaps. No renal calico-pyelic dilation. Blood chemistry tests were performed in the emergency room to assess complete blood count, coagulation, $\beta$-hCG, and clinical chemistry. No serious anaemia was found (Hb: $11.0 \mathrm{~g} / \mathrm{dl}$ ), a $\beta$-hCG of 54,000 $\mathrm{mIU} / \mathrm{ml}$, and coagulation and clinical chemistry were within limits.

It was decided to hospitalize the patient and to set up a therapy based on progesterone $1 \mathrm{fl}$ i.m. per day, hydroxyprogesterone caproate $1 \mathrm{fl}$ i.m. every 3 days, acetaminophen as needed, and intravenous fluids. The following morning, during the next blood chemistry check, haemoglobin was stable $(10.6 \mathrm{~g} / \mathrm{dl})$ and an increase in painful symptoms in the abdominal area was registered. It was thus decided to take the patient to the operating room after obtaining a specially drawn up informed consensus.

Levator palpebrae superioris surgery was performed for the elevated risk of tubal rupture and to save the intrauterine pregnancy. After preparation of the operating table, positioning of a Foley catheter, disinfection of the skin, and delimitation of the operating field with sterile sheets, an umbilical access with a Verres needle and 3 accessory routes was performed, 
after safety tests. Once the optic was introduced, the uterus, regular in appearance and in median position, was visualized. A modest blood layer was observed in the Douglas cavity, as well as a large blood clot in the left ovarian dimple, probably formed following fissuring of the ipsilateral tuba. The left tube appeared to be of regular morphology and dilated due to the presence of the ectopic pregnancy. The ipsilateral ovary was regular in appearance. The right tube and ovary appeared to be of regular morphology. The blood layer was drained, and the clot was aspirated. Left salpingectomy was then performed. Accurate control of haemostasis was achieved. The abdominal cavity was washed. At the control of the endoabdominal organs, they appeared unscathed. Trocars were removed under vision. The $\mathrm{CO}_{2}$ was desuffled and the incisions are sutured with absorbable thread. A transvaginal ultrasound was performed after the surgery, demonstrating the presence of foetal heartbeat into the uterus. The removed tissue was sent to the pathology laboratory for definitive histological examination. $1 \mathrm{fl}$. sc. Seleparin 0.4 for 7 days was added to the pre-set therapy after the surgery.

Two containers were sent to the pathology laboratory, the first of which contained the distal portion of the left tube of about $3 \mathrm{~cm}$, equipped with fimbriae, and the second contained a brownish clot with a maximum diameter of $3.2 \mathrm{~cm}$. On microscopic examination, the tubal sample appeared free from significant pathological alterations and generic subversions of the regular anatomy. In the context of the blood fibrin clot, some mononuclear and multinucleated trophoblastic elements were observed, rare villar structures, most of which had a hydropic appearance. Some villi also showed partial regressive phenomena. The pathologist confirmed the surgical suspicion of heterotopic pregnancy with ectopic implantation at the level of the left fallopian tube (Fig. 1, 2).

After the surgery the patient rested for 3 days at Cervello Hospital. Her vital parameters were carefully monitored 3 times per day, and 1 transvaginal ultrasound was performed once a day to monitor the uter-

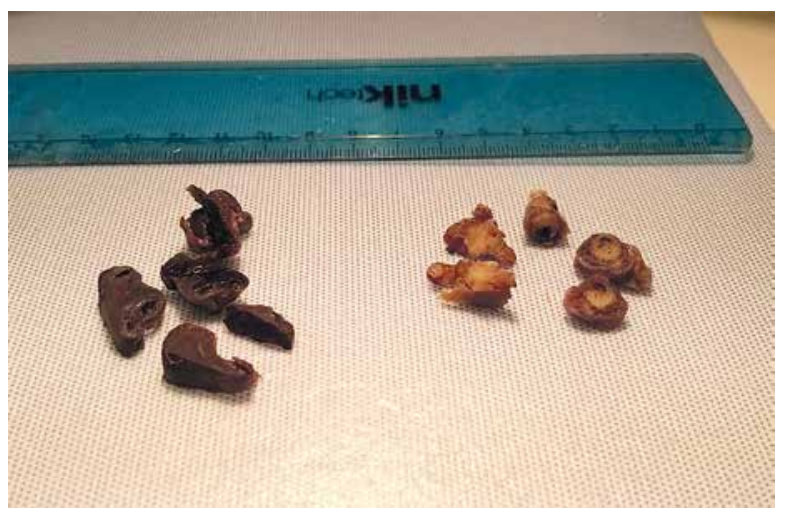

Fig. 1. Heterotopic pregnancy and left fallopian tube, after dissection ine pregnancy, demonstrating the presence of a foetal heartbeat.

After being discharged, the pregnancy was monitored by transvaginal ultrasound once a week for the first month, and on bi-weekly basis until the end of the first trimester of pregnancy. No anomalies were found. The patient was made aware of the benefits of the combined screening of the first trimester of pregnancy for the purpose of calculating the risk of foetal chromosomopathies and the risk of development of pre-eclampsia and accepted its execution. From the combination of the values resulting from the $\beta$-hCG, PAPP-A, and PIGF serum dosage and the ultrasound measurement of nuchal translucency, the pregnancy was classified as low risk. At $19+4$ weeks of amenorrhoea the patient underwent ultrasound screening of the II trimester of pregnancy, which demonstrated the absence of morphological and flow anomalies, regular amniotic fluid index, and regular growth of the foetus. At the time of writing this case report, the patient has performed her final obstetric visit and obstetric ultrasound at $34+5$ weeks of amenorrhoea. Regular foetal growth, normal amniotic fluid, and no abnormalities of flow or placental insertion were found. The pregnancy was followed-up until its end and assisted in carrying out the delivery. At the end of August 2021, the pregnancy ended with the birth of a living newborn $(3225 \mathrm{~g}, 48 \mathrm{~cm}$ ) with a score of 10/10 in the Apgar scale. However, due to cephalic posterior and deflexed position, a caesarean section was necessary.

\section{Conclusions}

A high index of suspicion is required to identify heterotopic pregnancies. Owing to its variable presentation and high mortality. All gynaecologists must consider heterotopic pregnancy as a possible diagnosis in a pregnant patient with abdominal pain and/or vaginal bleeding. Transvaginal ultrasound and early surgical intervention are key to avoid adverse outcomes and save the intrauterine pregnancy.

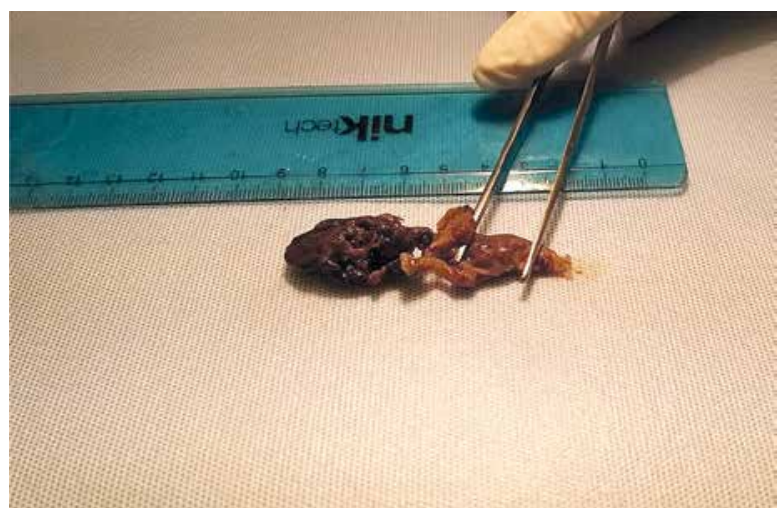

Fig. 2. Heterotopic pregnancy and left fallopian tube, before dissection 


\section{Disclosure}

The authors report no conflict of interest.

\section{References}

1. Baggio S, Garzon S, Russo A, et al. Fertility and reproductive outcome after tubal ectopic pregnancy: comparison among methotrexate, surgery and expectant management. Arch Gynecol Obstet 2021; 303: 259-268.

2. Wang ET, Kathiresan ASO, Bresee $C$, et al. Abnormal implantation after fresh and frozen in vitro fertilization cycles. Fertil Steril 2017; 107: 1153-1158.

3. Clayton HB, Schieve LA, Peterson HB, et al. A comparison of heterotopic and intrauterine-only pregnancy outcomes after assisted reproductive technologies in the United States from 1999 to 2002. Fertil Steril 2007; 87: 303-309.

4. Santoro G, Laganà AS, Sturlese E, Giacobbe V, Retto A, Palmara V. Developmental and clinical overview of lithopaidion. Gynecol Obstet Invest 2014; 78: 213-223.

5. Serpil T, Cihan K, Levent Y, Murat E. Spontaneous heterotopic pregnancy causing tubal rupture in a patient with intrauterine device in-situ. BTDMJB 2014; 10: 121-123.

6. Cozzolino M, Vitagliano A, di Giovanni MV, et al. Ultrasound-guided embryo transfer: summary of the evidence and new perspectives. A systematic review and meta-analysis. Reprod Biomed Online 2018; 36: 524-542.

7. Garzon S, Laganà AS, Pomini P, Raffaelli R, Ghezzi F, Franchi M. Laparoscopic reversible occlusion of uterine arteries and cornuostomy for advanced interstitial pregnancy. Minim Invasive Ther Allied Technol 2019; 28: 359-362.

8. Di Spiezio Sardo A, Vieira MDC, Laganà AS, et al. Combined systemic and hysteroscopic intra-amniotic injection of methotrexate associated with hysteroscopic resection for cervical pregnancy: a cutting-edge approach for an uncommon condition. Eurasian J Med 2017; 49: 66-68.

9. Hassani K, Bouazzaoui A, Khatouf M, Mazaz K. Heterotopic pregnancy: a diagnosis we should suspect more often. J Emerg Trauma Shock 2010; 3: 304.

10. Guan Y, Ma C. Clinical outcomes of patients with heterotopic pregnancy after surgical treatment. J Minim Invasive Gynecol 2017; 24: 1111-1115.

11. Lagana AS, Vitale SG, de Dominici R, et al. Fertility outcome after laparoscopic salpingostomy or salpingectomy for tubal ectopic pregnancy a 12-years retrospective cohort study. Ann Ital Chir 2016; 87: 461-465.

12. Doğan K, Kaya C, Ilıman DE, Karaca I, Cengiz H. A spontaneous heterotopic pregnancy presenting with acute abdomen treated with natural orifice transluminal endoscopic surgery procedure: case report. Turk J Obstet Gynecol 2019; 16: 271-273. 